Article

\title{
Passive Coagulability Assay Based on Coherence-Gated Light Scattering
}

\author{
Mahed Batarseh ${ }^{1}$, Jose Rafael Guzman-Sepulveda ${ }^{1,+}{ }^{\mathbb{C}}$, Ruitao $\mathrm{Wu}^{1}{ }^{1}$, \\ William M. DeCampli ${ }^{2,3}$ and Aristide Dogariu ${ }^{1, *}$ \\ 1 CREOL, the College of Optics and Photonics, University of Central Florida, Orlando, FL 328016, USA; \\ mahedbatarseh@knights.ucf.edu (M.B.); r.guzman@knights.ucf.edu (J.R.G.-S.); \\ ruitao.wu@knights.ucf.edu (R.W.) \\ 2 Pediatric Cardiothoracic Surgery, the Heart Center, Arnold Palmer Hospital for Children, \\ Orlando, FL 32806, USA; William.Decampli@orlandohealth.com \\ 3 College of Medicine, University of Central Florida, Orlando, FL 32816, USA \\ * Correspondence: adogariu@creol.ucf.edu; Tel.: +1-407-823-6839 \\ + Currently at the Center for Research and Advanced Studies of the National Polytechnic Institute \\ (CINVESTAV Unidad Monterrey), Apodaca, Nuevo Leon 66600, Mexico.
}

Received: 22 September 2020; Accepted: 16 October 2020; Published: 20 October 2020

\begin{abstract}
Coagulation monitoring relies on in vitro tests where the clot formation is induced using external stimuli. We report an optical method capable of revealing the propensity of coagulation based solely on the natural dynamics of erythrocytes in whole blood. In contrast to traditional techniques, our approach provides means to assess the blood coagulability without the need to chemically trigger the coagulation. Results of correlations with standard clinical methods suggest that this optical assay could be used for continuous management of blood coagulation during clinical procedures.
\end{abstract}

Keywords: coagulation; low-coherence dynamic light scattering; coagulation assays

\section{Introduction}

Coagulation is a factor activation cascade process that results in the formation of a blood clot. It takes place both during hemostasis, the natural cessation of blood loss after a vessel is damaged, as well as in pathological thrombosis, where a thrombus forms inside a vessel [1,2]. Pathological thrombosis can lead to several major acute and chronic conditions including venous thromboembolism, cerebral stroke, myocardial infarction, pulmonary embolism, and necrosis, among others [3]. Different coagulation assays are commonly used to monitor and diagnose these conditions.

Assessing the coagulation status of blood is also critical for managing the blood condition during a variety of clinical procedures. These include cardiovascular surgeries requiring cardiopulmonary bypass $(\mathrm{CPB})$, major surgeries in patients with coagulation disorders, extracorporeal life support (ECLS, ECMO), catheter-based interventional procedures, and hemodialysis, among others [4]. In these situations, a fast assessment of the coagulation status of blood is highly desirable.

The current state of the art in coagulation monitoring relies on provocative, end-point, non-physiologic in vitro tests, where clot formation is induced using external stimuli such as kaolin, glass beads, or calcium [5]. These clot-based tests can be performed on whole blood, e.g., activated clotting time (ACT) and thromboelastography (TEG) or on citrated plasma, e.g., prothrombin time (PT) and activated partial thromboplastin time (aPTT). In general, they are time-consuming because of the sample preparation requirements and the need for full coagulation to occur before global features of the coagulation process are determined. In some cases, chromogenic coagulation assays are also used to measure the activity of specific single factors $[6,7]$. 
Ultimately, the choice of a coagulation test is determined by both the level of detail desired and the other particular constraints of the measurement situation [5,8].

Due to its simplicity, ACT is one of the gold standards in point-of-care testing (POCT). The small volume of blood required and the minimal sample handling makes it useful in various clinical settings involving the administration of anticoagulants where the analytical range of other tests, such as aPTT and anti-Xa, is exceeded. The ACT test is an end-point measurement of the time it takes for whole blood to clot after an activator is administered, starting from a liquid state [9]. During the assay, after an activator is introduced, the coagulation process evolves under "accelerated" conditions and the propensity of blood to coagulate in its initial state (coagulability) is then associated with the time necessary for the sample to clot.

Thromboelastrometry and thromboelastography provide a more detailed evaluation of the dynamic properties of whole blood during coagulation, and are used in POCT. TEG relies on the rotation of a pin submerged in an oscillating cup of blood to determine the global viscoelastic properties and the clot strength [10]. Frequently, this measurement is also performed under "accelerated" conditions by using coagulation activators in order to reduce the duration of the measurement down to reasonable time frames [11-13]. The process of clot formation is described by four parameters: the reaction time (R) for fibrin polymer generation (initiation stage), the coagulation time $(\mathrm{K})$ taken to achieve a certain level of clot strength (amplification stage), the angle ( $\alpha \_$angle) which measures the speed at which fibrin builds up and cross-linking occurs (propagation phase), and the maximum amplitude (MA) representing the ultimate clot strength (end of the coagulation stage). Using instrument-specific coefficients, these four parameters can also be linearly combined into a so-called coagulation index (CI) [10].

Recently, we demonstrated a new way to monitor the blood conditions without relying on induced coagulation. The Low-Coherence Dynamic Light Scattering (LC-DLS) technique provides real-time information about the blood status as determined by its microscopic viscoelastic properties. This is in contrast to ACT and TEG assays that rely on examining the coagulation process until a solid clot is formed. A pilot study on ten patients has indicated that (i) the LC-DLS technique has the necessary sensitivity to discriminate between different conditions of blood coagulability and (ii) the information retrieved significantly correlates with ACT [14].

Here we extend the LC-DLS study to a larger cohort of 30 patients and provide a comprehensive correlation analysis with measurements of ACT and TEG. Aside from confirming a strong correlation with both ACT and TEG, we provide insights into the dynamics of incipient coagulation under natural conditions, and the relation to blood properties at advanced stages of the process. The developing correlation between the real-time information provided by LC-DLS and the end-point results of the externally triggered coagulation demonstrate the efficacy of this technique to monitor passively the early changes in the status of blood as required for efficient blood management during clinical procedures.

\section{Materials and Method}

\section{LC-DLS Technique}

The LC-DLS operation relies on the temporal fluctuations of the light intensity scattered from whole blood. In the time scales accessible in LC-DLS, these fluctuations are due to the movement of erythrocytes, which act as independent, rigid Brownian probes for the mechanical properties of the complex fluid surrounding them. This is in fact a micro-rheology measurement, where the scattered intensity fluctuations can be used to measure properties of complex fluids passively, in the presence of intrinsic thermal forces alone [15-17].

It can be estimated that, at physiological conditions, the erythrocytes explore diffusively less than $1 \%$ of their own diameters during 1-10 ms. This means that in the frequency range 0.1 to $1 \mathrm{kHz}$, LC-DLS provides information about the viscoelasticity of medium in the immediate surroundings of an erythrocyte. The measurement is based on a robust setup where the light is sent out and detected through the same optical fiber element. More details can be found in the Supplementary Materials of 
Ref. [14]. Practically, the dynamic information is recovered from the temporal autocorrelation of the fluctuations of light intensity scattered or, alternatively, from the corresponding power spectral density (PSD) of these intensity fluctuations.

At short time scales, the information about the microenvironment is encoded in the average mean-square displacement (MSD) of the probes (erythrocytes). In fact, MSD provides a close representation to the complex fluid's response to an imposed shear strain $[18,19]$. In other words, the overall mechanical susceptibility of the fluid determines the way in which the probes diffuse. The process can be described as the MSD evolution $\left\langle\Delta \mathrm{r}^{2}(\tau)\right\rangle=\mathrm{D} \tau^{\alpha}$ with a constant diffusion coefficient $\mathrm{D}$ and a viscoelasticity-dependent coefficient $\alpha$. Depending on the value of this coefficient, the diffusion is classified as normal for $\alpha=1$, and as sub- or super-diffusion for $\alpha<1$ and $\alpha>1$, respectively [20,21]. Importantly, the effective parameter $\alpha$ determines the asymptotic PSD: $P(f)=\mathfrak{J}\left[\exp \left(-\frac{q^{2}}{6} \Delta r^{2}(\tau)\right)\right] \sim f^{-(\alpha+1)}[22,23]$. Thus, in the case of whole blood where the erythrocytes are the diffusing probes, the shape of $\mathrm{P}(\mathrm{f})$ depends on the viscoelasticity of the complex fluid consisting of plasma and macromolecules. Due to the complex protein interactions, this viscoelasticity is expected to change during the process of coagulation.

Typical LC-DLS measurements on whole blood taken at different times during the incipient, natural coagulation are illustrated in Figure 1. As can be seen, the shape of $P(f)$ evolves indicating a reduction in the value of $\alpha$, which relates to the slope of the PSD in the logarithmic representation as shown in Figure 1. This means that, as blood starts to coagulate, the Red blood cells (RBCs) diffusion becomes more sub-diffusive, which can be associated to an increasing elasticity of the surrounding viscoelastic fluid. We emphasize that in these measurements no coagulation activators are used. Thus, the variations observed in Figure 1 refer to minute changes in the mechanical properties of blood that take place during the incipient stages of natural coagulation, which is indicative of the high sensitivity of this optical measurement.

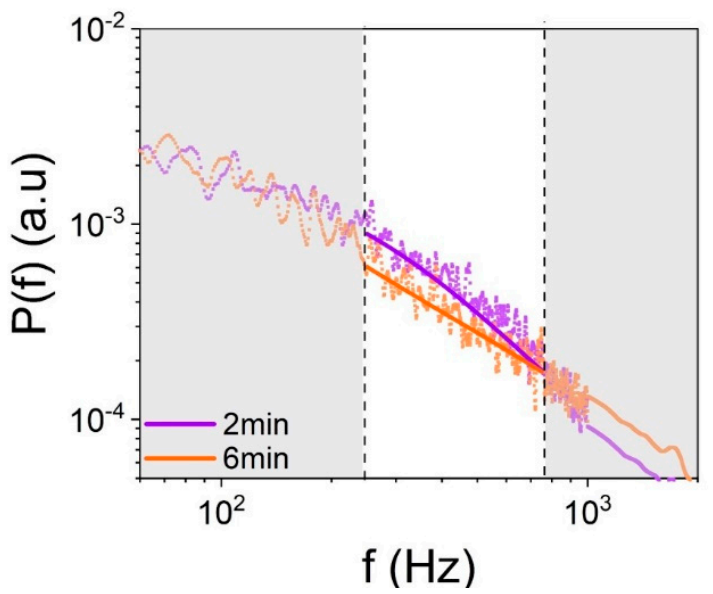

Figure 1. Typical Low-Coherence Dynamic Light Scattering (LC-DLS) power spectra measured on whole blood at two different times after the sample was drawn. The solid lines show the fitted $\mathrm{P}(\mathrm{f})$ in the band of interest $(250-750 \mathrm{~Hz})$, from where the logarithmic slope $\gamma$ is estimated. In time, the value of $\gamma$ reduces, which indicates the transition towards a more sub-diffusive motion of the RBCs. These measurements were performed during natural blood coagulation without external accelerators.

In the LC-DLS experiments, $\mathrm{P}(\mathrm{f})$ is measured in the frequency range from $1 \mathrm{~Hz}$ to $10 \mathrm{kHz}$, with $1 \mathrm{~Hz}$ resolution and an integration time of $30 \mathrm{~s}$. An averaged $\mathrm{P}(\mathrm{f})$ was obtained from four consecutive measurements, which means that one power spectrum, as indicated by the dotted lines in Figure 1, is obtained every $2 \mathrm{~min}$. Then, the averaged $\mathrm{P}(\mathrm{f})$ is fitted with a weighted superposition of Lorentzian functions, $P(f)=(2 / \pi) \sum_{\mathrm{i}=1}^{\mathrm{N}}\left(\mathrm{a}_{\mathrm{i}} / \tau_{\mathrm{i}}\right)\left[\mathrm{f}^{2}+\left(1 / \tau_{\mathrm{i}}\right)^{2}\right]^{-1}$ with different relative amplitudes $a_{i}$ and relaxation times $\tau_{i}$. This decomposition into Lorentzian modes relies on the 
rigorous single-scattering treatment for a monodisperse collection of particles whose diffusive motion is uncorrelated [22,24]. Besides its physical meaning, this fitting procedure provides means to generate a smoother PSD appropriate for data retrieval. We found that, for the entire set of measurements, five Lorentzians $(N=5)$ is sufficient to follow the overall shape of $P(f)$ at all times over the frequency range $(1 \mathrm{~Hz}$ to $10 \mathrm{kHz})$, while keeping the original $1 \mathrm{~Hz}$ resolution. The local logarithmic slope of $\mathrm{P}(\mathrm{f})$ is then evaluated locally and an average value $\gamma=-(\alpha+1)$ is estimated in the frequency range from $250 \mathrm{~Hz}$ to $750 \mathrm{~Hz}$, as shown in Figure 1. The parameter $\gamma$ indicates the average decay of $\mathrm{P}(\mathrm{f})$ within a frequency band, which is much narrower than the frequency range of the fit. As mentioned earlier, $\gamma$ quantifies practically the type of erythrocytes diffusion, which, in turn, is determined by the microscopic viscoelasticity of plasma. The range of $\gamma$ lies between the limits of viscous diffusion $(\gamma=-2)$ and elastic confinement $(\gamma=-1)$.

As an example of LC-DLS capabilities, let us now examine the initial stages of coagulation of blood with different coagulability. Figure 2 illustrates how the measurement clearly captures the different evolution of $\gamma$ for untreated and heparinzed blood samples of the same origin (from the same patient). In the case of untreated blood, one can clearly see a progressive reduction of the absolute value of the log slope $|\gamma|=\alpha+1$, which indicates that the RBCs' motion transitions gradually to a more sub-diffusive state characteristic to a more and more elastic environment. On the other hand, the thinning effect of heparin, administrated to the patient moments before the measurement, is to inhibit the creation of larger macromolecules responsible for the elastic behavior. The effect is evident: not only that coagulation is blocked but also there is still an ongoing action of heparin as apparent in the slight increase of $|\gamma|$. Remember that a value of $\gamma$ closer to -2 indicates that the environment surrounding the erythrocytes is more fluid ( $\gamma=-2$ corresponds to a purely Newtonian fluid). We would like to emphasize that the differences illustrated in Figure 2 were identified in the initial stages of blood evolution without the need of an external chemical trigger even from the beginning of the measurement.

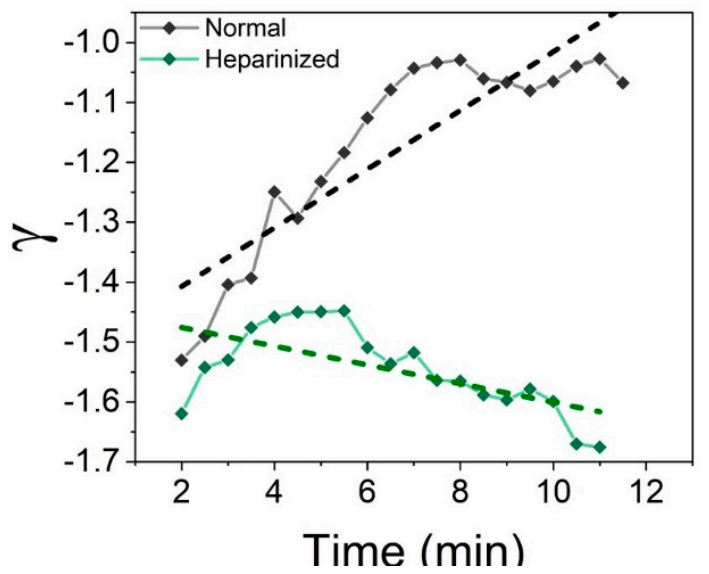

Figure 2. Evolution of logarithmic slope $\gamma$ in the range of $250-750 \mathrm{~Hz}$ for untreated and heparinized blood drawn from the same patient. The time is measured from when the sample was drawn (untreated blood) or prepared (heparin administration to the patient moments prior to blood draw). The coagulation evolves in normal conditions without any chemical trigger. A progressive reduction of $|\gamma|$ indicates a transition away from normal diffusion and towards a more elastic environment. Diffusion in purely viscous fluid corresponds to $\gamma=-2$.

\section{Results}

\subsection{Correlation with Coagulation Assays}

Having established some of the LC-DLS capabilities, we will now examine its outcome in comparison with the conventional coagulation tests ACT and TEG. Recall that the LC-DLS is a passive measurement where coagulation is not induced as opposed to the conventional assay. Thus, a solid clot 
is not formed; only incipient coagulation takes place as illustrated in Figure 3. However, a correlation between LC-DLS and the conventional assays can be expected because both measurements share some features that define the initial conditions. In this regard, a direct correlation between different measurements is the standard approach. Nevertheless, we have to anticipate the existence of inherent interpatient variability because the measurements involved access different properties at different scales (microenvironment versus global). This could hinder a direct association between raw measurements. Moreover, it is well known that the patients' response to the same dose of anticoagulant can vary significantly. To mitigate the influence of such potential inconsistencies, we also implemented a differential correlation analysis as a way to normalize individual patients to themselves. In this approach, the correlation is examined for differences between measurements that correspond to different conditions of blood drawn from the same patient. In other words, when comparing the outcomes of two different measurements $X=\{x\}$ and $Y=\{y\}$, one can examine a direct correlation $\mathrm{C}(\mathrm{x}, \mathrm{y})$ or a differential one $\mathrm{C}_{\Delta}(\Delta \mathrm{x}, \Delta \mathrm{y})$ where $\Delta \mathrm{x}=\mathrm{x}_{2}-\mathrm{x}_{1}$ and $\Delta \mathrm{y}=\mathrm{y}_{2}-\mathrm{y}_{1}$. In our particular case, a differential analysis is expected to normalize to a certain degree the effect of anticoagulant to the specific response of each individual patient.

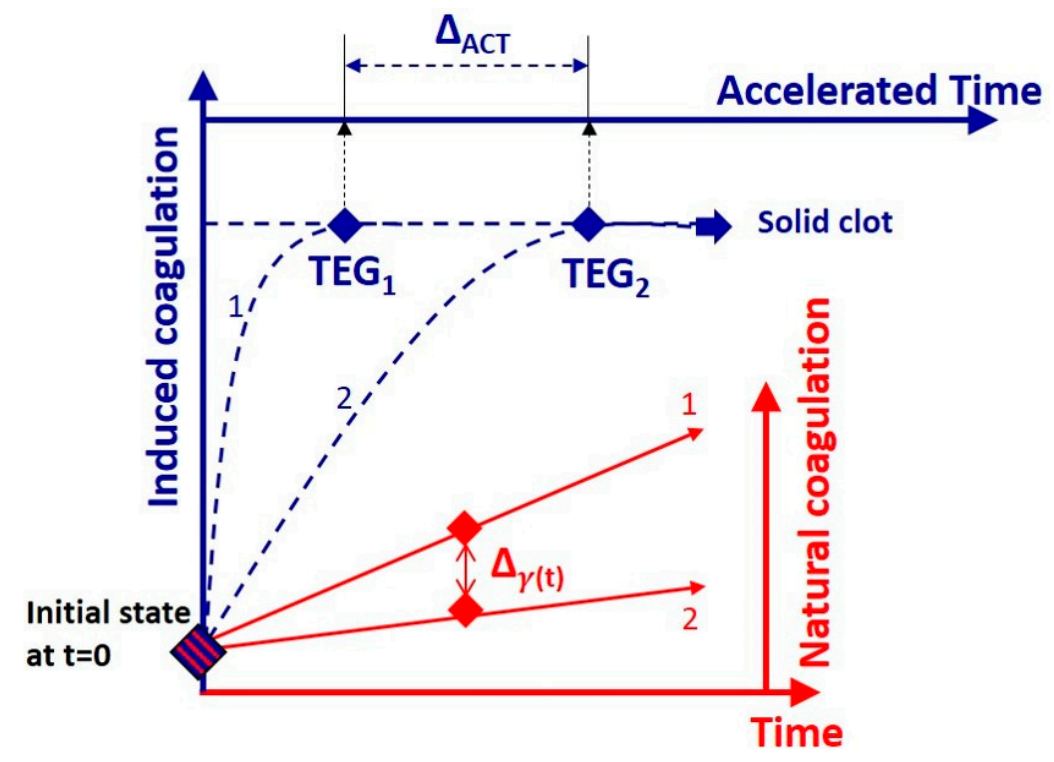

Figure 3. Comparison between the discrete results of end-point assays activated clotting time (ACT) and thromboelastography (TEG) and the continuous LC-DLS measure $\gamma(\mathrm{t})$. Untreated (1) and heparinized (2) blood evolution is natural (red) for LC-DLS or accelerated by an external trigger (blue) for ACT and TEG. Note that the differential measurements $\Delta_{\mathrm{ACT}}=\mathrm{ACT}_{2}-\mathrm{ACT}_{1}$ can only be evaluated between final clot states indicated by the blue diamonds.

A schematic comparison between these two approaches is illustrated in Figure 3. Note that, in the case of ACT and TEG assays, the coagulation process is artificially accelerated as opposed to its slower, natural evolution during the LC-DLS measurements. We emphasize that, in contrast to ACT and TEG, the LC-DLS is a real time continuous measurement where the status of the blood is monitored continuously as it evolves. Therefore, the correlation between the outcomes of these different experiments can be examined dynamically in terms of either $\gamma_{i}(t)$, or $\Delta_{\gamma}(t)=\gamma_{2}(t)-\gamma_{1}(t)$. It is expected that the time evolution of this correlation with the end-point results of ACT or TEG can reveal details of the coagulation process, as it will be shown later.

The correlation was assessed by a linear regression analysis at all times. The statistical significance was characterized by the evolution of descriptors of the linear regression, namely the slope of the linear model $(\beta)$, the Pearson's coefficient $(\mathrm{r})$, and the $p$-value $(p)$. Our systematic study was conducted in a clinical setting involving cardiovascular surgeries requiring cardiopulmonary bypass (CPB). This study was approved by both the University of Central Florida and the Orlando Health Institutional Review 
Boards. We obtained informed consent from all patients. For each patient, we used four blood samples, two taken before and two taken after the CPB procedure. The first sample collected before $\mathrm{CPB}$ was untreated blood at baseline conditions and the other one was blood after the full systemic anticoagulation treatment with heparin. Immediately after the $\mathrm{CPB}$, another sample of heparinized blood was taken. Finally, the last blood sample collected was after the effect of heparin had been reversed by the administration of protamine. Measurements of ACT, TEG, and LC-DLS were performed on all samples.

As mentioned before, the LC-DLS provides real-time information. However, for studying these correlations, we used a sliding window procedure where a block of four spectra were averaged to extract each $\gamma$ value and then translated in steps of $30 \mathrm{~s}$. The total time available for the LC-DLS measurements depends on the particular surgery protocol, which varies from patient to patient. Therefore, to study the correlations based on the same number of data points, the evolution of $\gamma(t)$ is approximated by its linear trend as indicated by the dashed lines in Figure 2.

\subsection{ACT Correlation}

Let us first examine the direct correlation between the ACT assay and the LC-DLS data, i.e., $C_{i}(t)=C\left(A C T_{i}, \gamma_{i}(t)\right)$ where the indices $i=1,2$ indicate blood in untreated or heparinized conditions. A measurement on heparinized blood $\mathrm{ACT}_{2}$ usually leads to a longer $\mathrm{ACT}$ time compared to untreated blood, either untreated or anticoagulant-reversed, and to a more negative value of $\gamma$ (closer to -2) in the LC-DLS measurement. Thus, a possible correlation between $\gamma$ and ACT is expected to be a decaying function. This trend is actually observed as indicated by the red line in Figure 4a. However, the direct evaluation of the statistical significance is hindered by the fact that, in cardiac surgeries, a large dose of heparin is usually administered. As a result, the ACT values cluster in two distinct modes corresponding to untreated and heparinized conditions as shown in Figure $4 \mathrm{~b}$, which affects the quality of the statistical comparison between ACT and LC-DLS results.
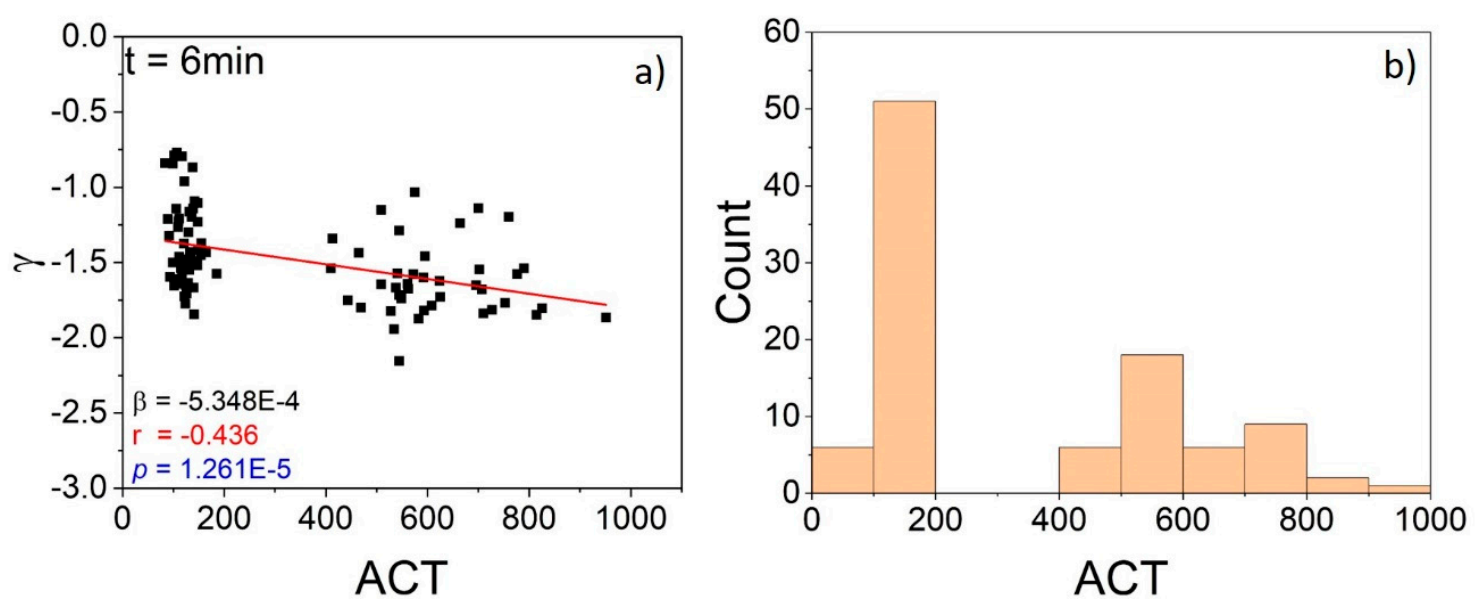

Figure 4. Correlation between $\gamma$ and ACT values. (a) Correlation after $6 \mathrm{~min}$ from the beginning of the LC-DLS measurement. (b) The distribution of ACT readings across the patient cohort (saturated ACT values (999) were removed from the data).

The situation improves when we examine the correlation between differential measurements. Note that, in this case, a large positive value of $\Delta_{\mathrm{ACT}}=\mathrm{ACT}_{2}-\mathrm{ACT}_{1}$ indicates a transition from a state of normal coagulation to a strong anti-coagulated state while a large negative value specifies the anticoagulant reversal. In the LC-DLS measurements, these transitions correspond to negative and positive values of $\Delta_{\gamma}$, respectively. In the context of RBS movement, these situations correspond to a condition closer to the normal diffusion of the RBCs (anticoagulated blood) or back to a more sub-diffusive (elastic) regime, respectively. Thus, one should also expect a negative correlation between 
$\Delta_{\mathrm{ACT}}$ and $\Delta_{\gamma}$. This is indeed the case as demonstrated by the results summarized in Figure $5 \mathrm{a}$. Note also that the $p$-value is orders of magnitude lower than in Figure 4a.
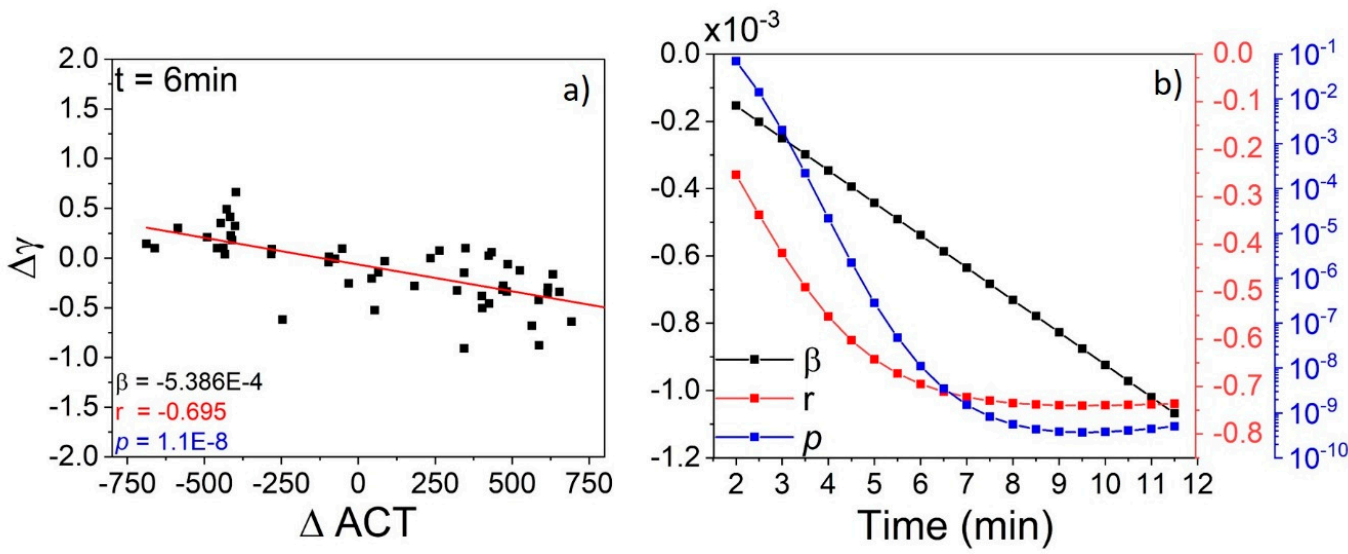

Figure 5. Correlation between $\Delta_{\mathrm{ACT}}$ and $\Delta_{\gamma}(\mathrm{t})$. (a) Correlation between $\Delta_{\mathrm{ACT}}$ and $\Delta_{\gamma}$ after $6 \mathrm{~min}$ from the beginning of the LC-DLS measurement. (b) Evolution of the non-stationary correlation in terms of the statistical parameters of the linear regression: the slope of linear model $(\beta)$, the Pearson's coefficient $(\mathrm{r})$, and the -value $(p)$. The results are averages over the 30 patients included in the study. For the differential correlation approach, the difference in $\Delta_{\mathrm{ACT}}$, and $\Delta_{\gamma}(\mathrm{t})$ is performed between consecutive measurements, and any differences associated to saturated ACT measurements (999) were removed from the pool of data.

More insights into the meaning of LC-DLS information can be gained by examining the time evolution of the correlation with ACT readings. The main characteristics of the non-stationary correlation between $\Delta_{\mathrm{ACT}}$ and $\Delta_{\gamma}(\mathrm{t})$ are shown in Figure $5 \mathrm{~b}$. The data is summarized over the entire cohort of 30 patients and averages the descriptors of many linear regressions like the one shown in Figure 5a. As can be seen, at early times and in the absence of a coagulation inducer, blood exhibits negligible coagulation; as a result, $\Delta_{\gamma}$ has very small values, which results in a weak correlation with $\Delta_{\mathrm{ACT}}$. However, as time passes and the incipient natural coagulation starts to develop, the correlation grows stronger as indicated by the increasing magnitudes of both $\beta$ and $r$, and also by the decrease of associated $p$-values.

Note that, despite the relatively weak correlation at early times (small value of $r$ ), $p$ remains small in all cases, which indicates the statistical significance of the linear regression. In other words, a linear model suffices to describe the relation between $\Delta_{\mathrm{ACT}}$ and $\Delta_{\gamma}(\mathrm{t})$ at all times during the evolution, even though the data points are sparse. Also, as seen in Figure $5 b$, the correlation between $\Delta_{\mathrm{ACT}}$ and $\Delta_{\gamma}(\mathrm{t})$ increases as the natural coagulation process starts to take place. After approximatively $6 \mathrm{~min}$, the value of $r$ saturates in spite of the fact that $\beta$ keeps showing a steeper and steeper slope. This means that strength of the correlation between $\Delta_{\mathrm{ACT}}$ and $\Delta_{\gamma}(\mathrm{t})$ can be only as strong as the saturation value and it is limited by the sparsity of the data points, which arise from the inter-patient variability. After $6 \mathrm{~min}$, a strong, statistically significant correlation with $\mathrm{r}=-0.73$ and $p<0.0001$ is reached as clearly seen in Figure 5b.

\subsection{TEG Correlation}

We have also examined the correlations with various parameters provided in the TEG assay. In this case, the values of $\gamma$ closer to -2 in the LC-DLS measurement corresponding to anti-coagulated states correspond to longer values for R and $\mathrm{K}$; smaller values of $\alpha \_$angle and MA; and more negative values of CI [12]. Thus, one expects to have negative correlations between $\gamma$ and both $\mathrm{R}$ and $\mathrm{K}$, and a positive correlation with the $\alpha \_$angle, MA, and CI, respectively.

A similar analysis was carried out for all the TEG parameters but Figure 6a illustrates only the correlation between $\mathrm{CI}$ and $\gamma$ measured at $6 \mathrm{~min}$ into the LC-DLS measurement. The expected positive 
correlation with the $\mathrm{CI}$ is clearly demonstrated. In Figure $6 \mathrm{~b}$, we summarize the time evolution of the linear regression descriptors $\beta, \mathrm{r}$, and $p$. The non-stationarity correlation is also observed in the TEG assay, indicating that as time passes and incipient natural coagulation starts to occur, the correlation becomes stronger and is confirmed by the increasing magnitude of $|\beta|$ and $|r|$, and the decrease of $p$. We note that the correlation between $\gamma$ and $\mathrm{CI}$ is the strongest (largest $|\mathrm{r}|$ ) in comparison with all other TEG parameters shown in the Appendix A. In contrast to the ACT assay, the correlation with the TEG was observed with the direct approach and the differential approach as shown in Appendix A. As mentioned earlier, a correlation between LC-DLS and the different TEG parameters is expected. However, it can also be expected that not all the TEG parameters have a strong association with the LC-DLS because a solid clot is never formed in the latter. This is verified, for instance, by the fact that the TEG-MA, which relates to the clot strength, has the weakest correlation with LC-DLS (Figures A1 and A2 in the Appendix A).
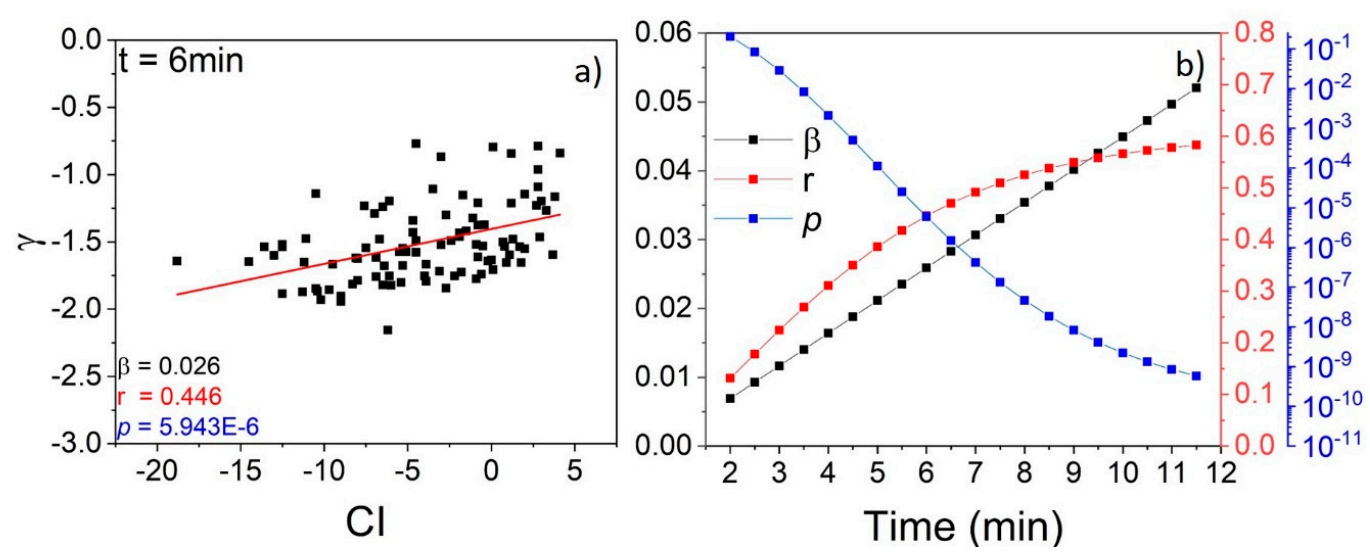

Figure 6. Correlation between coagulation index (CI) from the TEG assay and $\gamma(\mathrm{t})$. (a) Correlation between CI and $\gamma$ after 6 min from the beginning of the LC-DLS measurement. (b) Evolution of the non-stationary correlation in terms of the statistical parameters of the linear regression: the slope of linear model $(\beta)$, the Pearson's coefficient $(\mathrm{r})$, and the $p$-value $(p)$. The results are averages over the 30 patients included in the study.

These correlation studies between the LC-DLS and the conventional medical assays not only demonstrated that correlations indeed exist between these passive and induced measurements, but we also reveal how this correlation evolves when the process of natural coagulation progresses.

It is well known that correlations between different conventional assays are relatively weak, especially in the case when extracorporeal life support is involved. For instance, in [25] it was observed that most TEG parameters correlate weakly with both ACT and APTT. In the case of TEG-ACT, $r$ values in the range $0.29-0.35$ were obtained, with $p$-values of $0.003-0.054$. In the case of TEG-APTT, the largest correlation observed was for the TEG R time $(r=0.31, p=0.008)$. Interestingly, the correlation between ACT and platelet count was negligible. The best results in that study [25] were the moderated correlations observed between ACT-APTT $(r=0.56, p<0.001)$, and between the TEG-MA and the platelet count $(r=0.40, p<0.001)$. In another study [26], similar weak correlations were observed between ACT and heparin concentration, in infants undergoing CPB. Interestingly, they also noted some indications of a non-stationary correlation. Specifically, their correlation was negligible three minutes after systemic anticoagulation with heparin (ACT-Hepcon: $r=0.13, p=0.42$ ), but it became stronger by the end of the CPB (ACT-Hepcon: $r=0.3, p=0.05$ ). These examples create the context for understanding and interpreting the present results. In our study, the correlations are weak at earlier times but comparable with those reported in the literature. However, after only a few minutes the correlations grow stronger and become statistically significant indicating an increasing similarity between natural and induced aggregation processes. 


\section{Conclusions}

The results of this study demonstrate that a continuous dynamic light scattering measurement is able to discriminate between subtle differences in the initial coagulation stages of blood in untreated and heparinized states without any coagulation stimulus. We demonstrated that the results of LC-DLS measurements at early times correlate with traditional ACT and TEG data. These correlations between the results of different types of measurements are significant for two reasons. First, they compare an instantaneous property of blood with characteristics derived from observing an entire process until it ends in a clot. Second, the processes examined evolve at different paces: one progresses naturally and the other is accelerated by external stimuli. Unsurprisingly, the correlation with traditional assays are non-stationary in nature: it grows in time. We would like to emphasize that the approximately six minutes needed to observe a significant correlation with the medical assay is due to both the different nature of the measurements (continuous vs. end-point) and the finite time necessary for the heparin effect to set in. This is not a limitation of the LC-DLS technique, which infers the blood status continuously and discriminates between different blood conditions even at early times.

The chosen figure of merit, the slope $\gamma$ of the power spectrum of intensity fluctuations, is a just global parameter describing the viscoelastic properties of the fluid surrounding the erythrocytes. A more detailed model is necessary to relate the viscoelasticity to the microscopic composition of blood. In fact, LC-DLS provides the large-range frequency information necessary to describe fully the viscoelastic properties. Nevertheless, as our results demonstrate here, the slope $\gamma$ captures well the propensity of blood to coagulate. Most importantly, this parameter is retrieved from a measurement that is fully passive.

Our results strongly suggest the LC-DLS is able to monitor continuously the status of blood, especially in situations where a sensitive fast measurement is needed. The figure of merit $\gamma$ used here can report small variations in the status of blood during full systemic anti-coagulation where ACT is mostly insensitive and in much shorter time compared to TEG [27].

Author Contributions: A.D. and W.M.D., planned the project. A.D., designed and directed the research, and contributed to data analysis. W.M.D., directed the clinical research and contributed to data analysis. A.D. and J.R.G.-S., designed the experimental technique. J.R.G.-S., M.B. and R.W., contributed to data collection and analysis of the data. All authors contributed to writing the article. All authors have read and agreed to the published version of the manuscript.

Funding: This work was partially supported by the National Institutes of Health and the National Heart, Lung and Blood Institute under grant number 5R21HL124486.

Conflicts of Interest: The authors declare no conflict of interest. The funders had no role in the design of the study; in the collection, analyses, or interpretation of data; in the writing of the manuscript, or in the decision to publish the results

\section{Appendix A}

Appendix A.1. The Direct Correlation with All the TEG Parameters
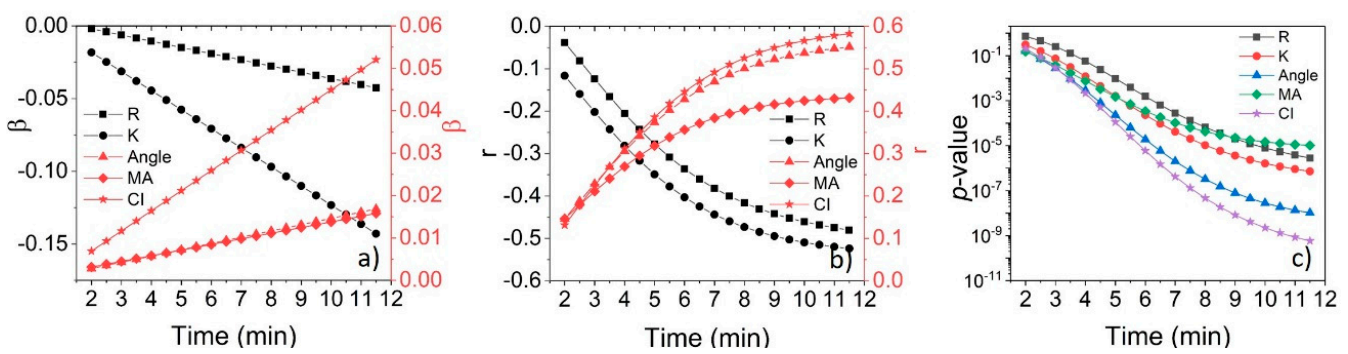

Figure A1. Summary of the evolution of the non-stationary correlation (between TEG parameters $(R, K$, Angle, $M A$, and $C I$ ) and $\gamma(t))$ in terms of the relevant parameters of the linear regression analysis based on the direct approach; (a) the slope of the linear model $(\beta),(\mathbf{b})$ the Pearson's coefficient $(\mathrm{r})$, and (c) the $p$-value $(p)$. The results shown are for the 30 patients included in our study. 


\section{Appendix A.2. The Differential Correlations with All the TEG Parameters}

In the differential analysis between two states for the TEG assay parameters, a transition from a state of normal coagulation to a state of anti-coagulation prolongs time reported in the $R_{2}$ and $K_{2}$ parameters and a decrease of values in angle, $\mathrm{MA}_{2}$, and $\mathrm{CI}_{2}$. This transition results in a large positive value of $\Delta_{\mathrm{R}}$ and $\Delta_{\mathrm{K}}$ and a large negative value of $\Delta_{\text {angle }}, \Delta_{\mathrm{MA}}, \Delta_{\mathrm{CI}}$ which corresponds to a negative $\Delta_{\gamma}$ in the LC-DLS measurement. The change from an anti-coagulant state to anti-coagulant reversal will result in a positive $\Delta_{\gamma}$, and the opposite trends for the above TEG parameters.
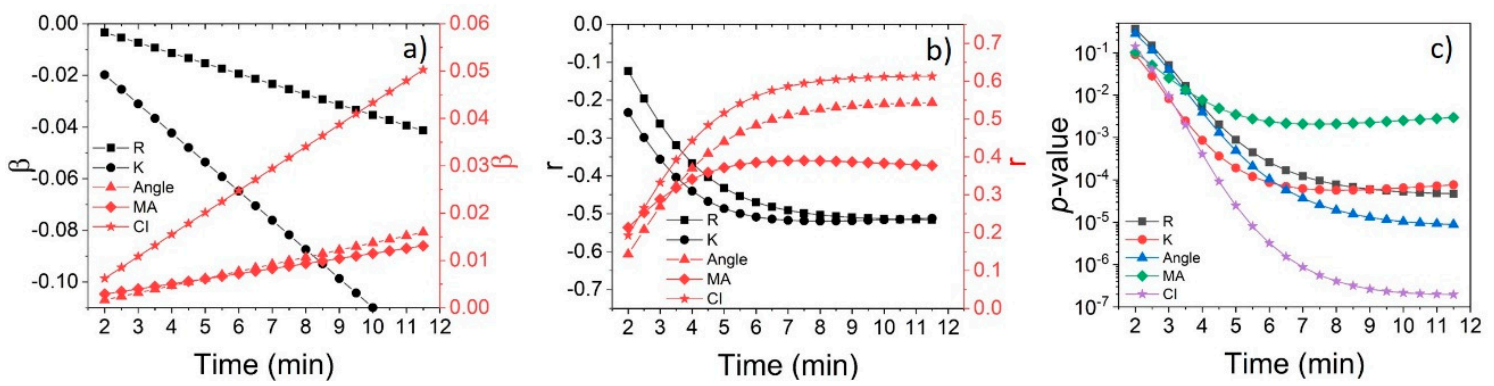

Figure A2. Summary of the evolution of the non-stationary correlation (between $\Delta_{\text {TEG }}$ parameters $\left(\Delta_{\mathrm{R}}, \Delta_{\mathrm{K}}, \Delta_{\text {Angle }}, \Delta_{\mathrm{MA}}\right.$, and $\left.\Delta_{\mathrm{CI}}\right)$ and $\left.\Delta_{\gamma}(\mathrm{t})\right)$ in terms of the relevant parameters of the linear regression analysis using the differential approach; (a) the slope of the linear model $(\beta)$, (b) the Pearson's coefficient (r), and (c) the $p$-value $(p)$. The results shown are for the 30 patients included in our study. For the differential correlation approach, the difference in $\Delta_{\mathrm{TEG}}$, and $\Delta_{\gamma}(\mathrm{t})$ is performed between consecutive measurements.

\section{References}

1. Smith, S.A.; Travers, R.J.; Morrissey, J.H. How it all starts: Initiation of the clotting cascade. Crit. Rev. Biochem. Mol. Biol. 2015, 50, 326-336. [CrossRef]

2. Palta, S.; Saroa, R.; Palta, A. Overview of the coagulation system. Indian J. Anaesth. 2014, 58, 515. [CrossRef]

3. Kumar, V.; Abbas, A.K.; Aster, J.C. Robbins Basic Pathology; Elsevier Health Sciences: Amsterdam, The Netherlands, 2017.

4. Funk, D.M. Coagulation assays and anticoagulant monitoring. In Hematology 2010, the American Society of Hematology Education Program Book; American Society of Hematology: Washington, DC, USA, 2012; Volume 2012, pp. 460-465.

5. Bates, S.M.; Weitz, J.I. Coagulation assays. Circulation 2005, 112, e53-e60. [CrossRef]

6. Barrowcliffe, T.W.; Raut, S.; Sands, D.; Hubbard, A.R. Coagulation and chromogenic assays of factor VIII activity: General aspects, standardization, and recommendations. In Seminars in Thrombosis and Hemostasis; Thieme Medical Publishers, Inc.: New York, NY, USA, 2002; pp. 247-256.

7. Potgieter, J.J.; Damgaard, M.; Hillarp, A. One-stage vs. chromogenic assays in haemophilia A. Eur. J. Haematol. 2015, 94, 38-44. [CrossRef] [PubMed]

8. Monagle, P. Haemostasis: Methods and Protocols; Humana Press: Totowa, NJ, USA, 2013.

9. Salzman, E.W.; Britten, A. Hemorrhage and Thrombosis: A Practical Clinical Guide; Little, Brown: Boston, MA, USA, 1965.

10. Bose, E.; Hravnak, M. Thromboelastography: A practice summary for nurse practitioners treating hemorrhage. J. Nurse Pract. 2015, 11, 702-709. [CrossRef]

11. Bowbrick, V.A.; Mikhailidis, D.P.; Stansby, G. The use of citrated whole blood in thromboelastography. Anesth. Analg. 2000, 90, 1086-1088. [CrossRef] [PubMed]

12. Da Luz, L.T.; Nascimento, B.; Rizoli, S. Thrombelastography (TEG $\left.{ }^{\circledR}\right)$ : Practical considerations on its clinical use in trauma resuscitation. Scand. J. Trauma Resusc. Emerg. Med. 2013, 21, 29. [CrossRef] [PubMed]

13. Evans, P.; Hawkins, K.; Lawrence, M.; Williams, R.; Barrow, M.; Thirumalai, N.; Williams, P. Rheometry and associated techniques for blood coagulation studies. Med. Eng. Phys. 2008, 30, 671-679. [CrossRef] [PubMed]

14. Guzman-Sepulveda, J.; Argueta-Morales, R.; DeCampli, W.; Dogariu, A. Real-time intraoperative monitoring of blood coagulability via coherence-gated light scattering. Nat. Biomed. Eng. 2017, 1, 1-6. [CrossRef] 
15. Popescu, G.; Dogariu, A. Dynamic light scattering in localized coherence volumes. Opt. Lett. 2001, 26, 551-553. [CrossRef] [PubMed]

16. Popescu, G.; Dogariu, A.; Rajagopalan, R. Spatially resolved microrheology using localized coherence volumes. Phys. Rev. E 2002, 65, 041504. [CrossRef] [PubMed]

17. Guzman-Sepulveda, J.; Dogariu, A. Probing complex dynamics with spatiotemporal coherence-gated DLS. Appl. Opt. 2019, 58, D76-D90. [CrossRef] [PubMed]

18. Mason, T.G. Estimating the viscoelastic moduli of complex fluids using the generalized Stokes-Einstein equation. Rheol. Acta 2000, 39, 371-378. [CrossRef]

19. Mason, T.G.; Weitz, D.A. Optical measurements of frequency-dependent linear viscoelastic moduli of complex fluids. Phys. Rev. Lett. 1995, 74, 1250. [CrossRef]

20. Metzler, R.; Jeon, J.-H.; Cherstvy, A.G.; Barkai, E. Anomalous diffusion models and their properties: Non-stationarity, non-ergodicity, and ageing at the centenary of single particle tracking. Phys. Chem. Chem. Phys. 2014, 16, 24128-24164. [CrossRef]

21. Vlahos, L.; Isliker, H.; Kominis, Y.; Hizanidis, K. Normal and anomalous diffusion: A tutorial. arXiv 2008, arXiv:0805.0419.

22. Sohn, I.; Rajagopalan, R.; Dogariu, A. Spatially resolved microrheology through a liquid/liquid interface. J. Colloid Interface Sci. 2004, 269, 503-513. [CrossRef]

23. Douglass, K.M.; Sukhov, S.; Dogariu, A. Superdiffusion in optically controlled active media. Nat. Photonics 2012, 6, 834-837. [CrossRef]

24. Berne, B.J.; Pecora, R. Dynamic Light Scattering: With Applications to Chemistry, Biology, and Physics; Courier Corporation: North Chelmsford, MA, USA, 2000.

25. Alexander, D.; Butt, W.; Best, J.; Donath, S.; Monagle, P.; Shekerdemian, L. Correlation of thromboelastography with standard tests of anticoagulation in paediatric patients receiving extracorporeal life support. Thromb. Res. 2010, 125, 387-392. [CrossRef]

26. Guzzetta, N.A.; Monitz, H.G.; Fernandez, J.D.; Fazlollah, T.M.; Knezevic, A.; Miller, B.E. Correlations between activated clotting time values and heparin concentration measurements in young infants undergoing cardiopulmonary bypass. Anesth. Analg. 2010, 111, 173-179. [CrossRef]

27. Ortmann, E.; Rubino, A.; Altemimi, B.; Collier, T.; Besser, M.; Klein, A. Validation of viscoelastic coagulation tests during cardiopulmonary bypass. J. Thromb. Haemost. 2015, 13, 1207-1216. [CrossRef] [PubMed]

Publisher's Note: MDPI stays neutral with regard to jurisdictional claims in published maps and institutional affiliations.

(C) 2020 by the authors. Licensee MDPI, Basel, Switzerland. This article is an open access article distributed under the terms and conditions of the Creative Commons Attribution (CC BY) license (http://creativecommons.org/licenses/by/4.0/). 